Originalien

Monatsschr Kinderheilkd 2022 · 170:435-442 https://doi.org/10.1007/s00112-021-01220-x Eingegangen: 13. Januar 2021

Angenommen: 19. März 2021

Online publiziert: 21. Juni 2021

(c) Der/die Autor(en) 2021

\section{Redaktion}

Berthold Koletzko, München

Thomas Lücke, Bochum

Ertan Mayatepek, Düsseldorf

Norbert Wagner, Aachen

Stefan Wirth, Wuppertal

Fred Zepp, Mainz

\author{
J. Hansen ${ }^{1} \cdot$ R. Hanewinkel' $\cdot$ M. Goecke ${ }^{2} \cdot$ M. Morgenstern' \\ ${ }^{1}$ Institut für Therapie- und Gesundheitsforschung, IFT-Nord gemeinnützige $\mathrm{GmbH}$, Kiel, Deutschland \\ ${ }^{2}$ Bundeszentrale für gesundheitliche Aufklärung, Köln, Deutschland
}

\title{
Prävention der exzessiven Mediennutzung im Kindes- und Jugendalter
}

Medien (JIM)-Studie bei 214 Minuten (min) (wochentags). Die 16- bis 17Jährigen wiesen mit 243 min täglicher Onlinenutzung die längste Dauer auf [4].

Internetbezogene Störungen werden als Verhaltenssüchte angesehen, die bisher nicht abschließend konzeptualisiert und operationalisiert sind [5]. Vulnerable Jugendliche, bei denen sich eine exzessiv-dysfunktionale Nutzung von digitalen Medien zeigt, verbringen übermäßig viel Zeit online, nutzen digitale Medien, um Gefühlszustände besser zu bewältigen, während andere Aktivitäten und soziale Kontakte in der realen Welt stattdessen vernachlässigt werden. Nicht jede Phase einer exzessiven Nutzung entwickelt sich jedoch zu einer Sucht, sie kann sich auch wieder normalisieren. Es gibt aber Hinweise darauf, dass bei internetbezogenen Störungen nicht von einem vorübergehenden Phänomen mit hoher Wahrscheinlichkeit für Spontanremissionen auszugehen ist [5]. Als Folge einer computerspiel- und internetbezogenen Störung können sich physische Probleme wie beispielsweise Haltungsschäden, Rückenschmerzen und Bewegungsmangel, zudem auch erhebliche Beeinträchtigungen in einem breiten Spektrum psychischer Funktionen zeigen - Aggressivität, Aufmerksamkeits-, soziale, aber auch Leistungsprobleme $[3,12]$. Exzessiv-dysfunktionales Computerspielen (auch „internet gaming disorder" [IGD]), ist als Störungsbild in der aktuellen Version des Diagnostic and
Statistical Manual of Mental Disorders-5 (DSM-5) in Sektion III enthalten und wird in der 2022 in Kraft tretenden International Statistical Classification of Diseases and Related Health Problems-11 (ICD-11) als Störung aufgrund von Suchtverhalten aufgenommen. In Deutschland sind schätzungsweise $8 \%$ der 12- bis 19-Jährigen von einer computerspiel-oder internetbezogenen Störung betroffen [11].

Um einen verantwortungsvollen Umgang mit Medien zu erlernen, müssen Kinder und Jugendliche an die Nutzung herangeführt und für die Gefahren eines problematischen Gebrauchs sensibilisiert werden. Programmen zur Prävention der übermäßigen Mediennutzung kommt eine tragende Rolle $\mathrm{zu}$, und es besteht wissenschaftlicher Konsens über die Notwendigkeit von Interventionen im Kindes- und Jugendalter [18]. Präventive Maßnahmen sollten dabei frühzeitig ansetzen [5]. Hinsichtlich computerspielbezogener Störungen zeigten sich Maßnahmen als wirksam, die bei substanzgebundener Abhängigkeit durchgeführt werden (z.B. ressourcenorientierte Primärprävention) [15].

In der Vergangenheit durchgeführte Evaluationen von schulbasierten Präventionsprogrammen zeigten dabei positive Wirkungen auf die Nutzungsdauer von digitalen Medien sowie auf problematisches Verhalten. Bonnaire et al. berichteten von positiven Effekten einer 90minütigen schulbasierten Sensibilisierungsmaßnahme an einer französischen nach Angaben der Jugend, Information, 
Stichprobe mit 434 Schüler*innen, die sich in einer geringeren Nutzungsdauer in der Interventionsgruppe im Vergleich zur Kontrolle und verringerten IGDRaten widerspiegelten [2]. Eine südkoreanische Studie zeigte eine Zunahme an Selbstkontrolle und Selbstwirksamkeit nach Teilnahme an einer Intervention und eine Verringerung der Internetabhängigkeit sowie der im Internet verbrachten Zeit bei Schüler*innen der Mittelstufe [20]. Positive Effekte eines 4-stufigen schulbasierten Programms zur Förderung der Medienkompetenz auf die Dauer von Computerspielen und auf exzessiv-dysfunktionales Verhalten berichteten Walther et al. in einer clusterrandomisierten Studie mit einer deutschsprachigen Stichprobe mit 2303 11- bis 13-Jährigen [19].

Angesichts des rasanten digitalen Wandels besteht Bedarf an aktuellen, flächendeckenden Präventionsprogrammen, die Kinder und Jugendliche für eine exzessive Mediennutzung sensibilisieren und ihnen helfen wahrzunehmen, welches Ausmaß die Nutzung in ihrem Alltag einnimmt. Im folgenden Beitrag werden die Ergebnisse der Begleitforschung eines schulbasierten Projekts zur Prävention der exzessiven Mediennutzung im Kindes -und Jugendalter („NetPiloten") berichtet. Das Programm ist im Rahmen der Kampagne „Ins Netz gehen" der Bundeszentrale für gesundheitliche Aufklärung (BZgA) im Jahr 2013 entwickelt worden. Die folgende Fragestellung steht im Fokus: Führt die Interventionsteilnahme zu einem höheren Wissen über negative Folgen exzessiver Mediennutzung (Effekte auf kognitiver Ebene) und zu seltener problematischem Mediennutzungsverhalten (Effekte auf Verhaltensebene) im Vergleich zur Nichtteilnahme?

\section{Studiendesign und Unter- suchungsmethoden}

Es wurde eine kontrollierte Studie mit passender Stichprobe im 1. Schulhalbjahr 2019/2020 an weiterführenden Schulen in Brandenburg, Nordrhein-Westfalen und Schleswig-Holstein durchgeführt. Die Interventionsgruppe bestand aus Schüler*innen, die in den beiden Schul- jahren zuvor an der Intervention „NetPiloten“ teilgenommen hatten; Schüler*innen ohne Interventionsteilnahme bildeten die Kontrollgruppe (KG). Beide Gruppen wurden einmalig mittels standardisiertem Online-Fragebogen befragt (Postbefragung).

Die erforderlichen ministeriellen Genehmigungen wurden von den Aufsichtsbehörden erteilt. Neben den rechtlichen Erfordernissen wurde außerdem das Votum der Ethikkommission der Deutschen Gesellschaft für Psychologie eingeholt (Votum: „ethisch unbedenklich“, Az. RH 052019).

\section{Beschreibung der Intervention}

Die „Peer-to-peer“-Intervention „NetPiloten" ist im Rahmen der BZgAKampagne „Ins Netz gehen“ zur Prävention der exzessiven Mediennutzung im Jugendalter im Jahr 2013 entwickelt und erprobt worden und wird seitdem kontinuierlich weiterentwickelt. Schulen mit Sekundarstufe I können kostenfrei teilnehmen. Ziele sind u.a. das Erhöhen der Kenntnis über negative Folgen exzessiver Computerspielund Internetnutzung in den Zielgruppen, Förderung der Reflexionsfähigkeit sowie Einstellungsänderung zu eigener Computerspiel- und Internetnutzung, Förderung der selbstkritischen Änderung des Nutzungsverhaltens von Computerspiel- und Internetangeboten und der Beratungsbereitschaft im Bedarfsfall [7]. Sechst- und Siebtklässler werden in Workshops (2-mal $90 \mathrm{~min})$ für das Thema sensibilisiert und zur Selbstreflexion angeregt. Die Durchführung der Workshops erfolgt durch Acht- bis Zehntklässler derselben Schule, die im Rahmen einer 4-tägigen Schulung zu "Net-Piloten“ ausgebildet wurden. Die Ausbildung wird von geschulten Multiplikator*innen des Programms (z. B. Suchtpräventionsfachkräfte) durchgeführt, die eine Fortbildung zur Schulung der "Net-Piloten“-Intervention absolviert hatten [8].

\section{Durchführung}

Interventionsschulen wurden zur Teilnahme an der Studie eingeladen. Eine
Kontaktaufnahme $\mathrm{zu}$ Kontrollschulen erfolgte in zufällig ausgewählten Regionen der beteiligten Bundesländer. Die Teilnahme an der Studie war für alle Beteiligten freiwillig. Eine Einwilligungserklärungen der Erziehungsberechtigten musste vorliegen. Die Klarnamen der Schüler*innen wurden im Rahmen der Befragung nicht erfasst.

Die Durchführung der Befragung dauerte in der Regel maximal $45 \mathrm{~min}$ und erfolgte mittels externer Datenerheber*innen. Die Befragung wurde auf Wunsch der Schule hin auch ohne Externe durchgeführt. In diesem Fall erhielten die Ansprechpartner ${ }^{*}$ innen in den Schulen im Vorwege Instruktionen zur Durchführung.

\section{Studienvariablen}

Erfasst wurden folgende soziodemografischen Merkmale: Alter, Geschlecht, besuchte Schulart, Herkunft, subjektiver sozioökonomischer Status [6] sowie subjektive Schulleistung. Das Persönlichkeitsmerkmal „sensation seeking“ [16] wurde mit 2 Items erhoben, die Persönlichkeitsstruktur wurde mittels des Big Five Inventory (BFI) [14] erfasst. Das Wissen über problematische Mediennutzung wurde mittels eines Wissensquiz sowie Fragen zu negativen Folgen exzessiver Nutzung erhoben. Die Mediennutzung wurde wochentags und an schulfreien Tagen in Stunden und Minuten, die problematische Nutzung mittels Compulsive Internet Use Scale (CIUS) erfasst [9]. Die detaillierte Darstellung der erfassten Merkmale ist im Zusatzmaterial online zu finden.

\section{Statistische Analysen}

Das Ziehen von „matched samples" trägt zur Verbesserung der Abschätzung kausaler Effekte durch Verringerung des Ungleichgewichts der Kovariaten zwischen Studienbedingungen bei [1]. Hierfür wurde mittels der "Coarsened-exactmatching"-Methode aus der Gesamtstichprobe der befragten Schüler*innen eine Substichprobe beider Studienbedingungen gezogen. Merkmale, die für das Matching herangezogen wurden, waren: Alter, Geschlecht, besuchte Schulart, 
Monatsschr Kinderheilkd 2022 · 170:435-442 https://doi.org/10.1007/s00112-021-01220-x

(c) Der/die Autor(en) 2021

\section{J. Hansen · R. Hanewinkel · M. Goecke · M. Morgenstern}

\section{Prävention der exzessiven Mediennutzung im Kindes- und Jugendalter}

\section{Zusammenfassung}

Hintergrund. Für Kinder und Jugendliche ist die Nutzung digitaler Medien ein zentraler Bestandteil der Freizeitgestaltung. Bei etwa $8 \%$ der 12- bis 17-Jährigen ist von einer computerspiel- oder internetbezogenen Störung auszugehen.

Ziel der Arbeit. Ziel war die Überprüfung der Wirksamkeit eines schulbasierten „Peerto-peer"-Programms zur Prävention der exzessiven Mediennutzung im Kindesund Jugendalter („Net-Piloten“). Das primärpräventive Programm wird Schulen mit Sekundarstufe I von der Bundeszentrale für gesundheitliche Aufklärung (BZgA) in Zusammenarbeit mit den Landesstellen für Suchtfragen kostenfrei zugänglich gemacht. Material und Methode. Die Wirksamkeit von "Net-Piloten" wurde in einer kontrollierten
Studie (1:1-Matching) überprüft. Die Datenerhebung erfolgte über einen OnlineFragebogen im 1. Schulhalbjahr 2019/2020, an der insgesamt 834 Kinder und Jugendliche teilnahmen (mittleres Alter: 12 Jahre). Die Interventionsgruppe nahm an einem von Peers durchgeführten Workshop zur Sensibilisierung für die Risiken von exzessiver Mediennutzung und zur Förderung der Selbstreflexion teil.

Ergebnisse. Schüler*innen der Interventionsbedingung verfügten über ein höheres Wissen über negative Konsequenzen exzessiver Mediennutzung und wiesen geringere Nutzungszeiten (Computerspieldauer $\mathrm{F}(1,832)=6,45, p=0,011)$ auf als die Vergleichsgruppe. Der Anteil an Schüler*innen mit problematischer Mediennutzung war nach "Net-Piloten"-Teilnahme geringer im Vergleich zur Kontrolle $(F(1,832)=14,76$, $p<0,001$ ).

Diskussion. Die Befunde stehen im Einklang mit früheren Studien zur Effektivität von schulbasierten Programmen zur Vermeidung der problematischen Mediennutzung. Sie deuten darauf hin, dass schulbasierte Programme einer exzessiv-dysfunktionalen Mediennutzung vorbeugen können.

\section{Schlüsselwörter}

Problematischer Medienkonsum · Computerspiele · Internet · Präventionsprogramm · Peerto-peer-Projekt

\section{Prevention of excessive media use in childhood and adolescence}

\section{Abstract}

Background. The use of digital media plays an important role in the leisure time of children and young people. However, it can be assumed that about $8 \%$ of young people aged 12-17 years old have a computer game or internet-related disorder.

Objective. The aim was to test the effectiveness of a school-based peer-to-peer program for the prevention of excessive gaming and internet use in childhood and adolescence ("net-pilots").

Material and methods. A controlled study with a matched sample (1:1 matching) was used to examine the effects of the prevention program. Data assessment was carried out with an online survey during the first half of the school year 2019/2020. A total of 834 children and adolescents with a mean age of 12 years took part in the study. The intervention group took part in a peer-topeer workshop for sensitization to the risks of excessive media use and to promote selfreflection.

Results. Schoolchildren who participated in the intervention had a greater knowledge of the negative consequences of excessive media use and spent less time with media than the comparison group $(\mathrm{F}(1.832)=6.45, p=0.011)$. Moreover, the proportion of schoolchildren with problematic media use was lower in the intervention group compared to the control group $(\mathrm{F}(1.832)=14.76, p<0.001)$.

Conclusion. The findings are consistent with previous studies on the effectiveness of school-based programs for preventing problematic media use. They suggest that school-based programs can prevent excessive and dysfunctional gaming and internet use.

\section{Keywords}

Problematic media use - Computer games . Internet · Prevention program - Peer to peer project
Migrationshintergrund und Bundesland (1:1-Matching).

Die CIUS-Items wurden aufsummiert und eine CIUS-Skala gebildet (Range 0 bis 54, Cronbachs $\alpha=0,86$ ). Höhere Werte deuten auf eine höhere Problembelastung hin. Mittels ChiQuadrat-Anpassungstests und einfaktorieller Varianzanalysen wurden Unterschiede in den Merkmalsausprägungen der Interventions- und Kontrollgruppe untersucht.

Zur Effektprüfung auf Wissensebene erfolgten Gruppenvergleiche im Rahmen von Chi-Quadrat-Tests; Unterschiede im Mediennutzungsverhalten wurden mittels einfaktorieller Varianzanalysen geprüft. Alle Auswertungen wurden mit der Software Stata v15.0 (StataCorp LLC, College Station, TX, USA) durchgeführt.

\section{Ergebnisse}

\section{Analysestichprobe}

Die Analysestichprobe wurde aus einem Datenpool von 3347 Schüler*innen gezogen. Durch das Anwenden der 1:1-
Matching-Methode erhielten 417 Schüler*innen der Interventionsbedingung einen exakten Match in der Kontrollbedingung (die gematchte Person aus der Kontrollbedingung gleicht der Person aus der Interventionsbedingung in den Merkmalen Alter, Geschlecht, besuchte Schulart, Migrationshintergrund und Bundesland). Die Analysestichprobe umfasst somit 834 Schüler*innen mit einer Gleichverteilung auf die Studienbedingungen und einem mittleren Alter von 12,4 Jahren. Einen Überblick über die Merkmale der Analysestichprobe, 


\begin{tabular}{|c|c|c|c|}
\hline Merkmal & Kontrollgruppe & Interventionsgruppe & $p$ \\
\hline$n$ & 417 & 417 & $>0,999$ \\
\hline Alter in Jahren (M, SD) & $12,4( \pm 1,31)$ & $12,4( \pm 1,31)$ & $>0,999$ \\
\hline $\begin{array}{l}\text { Geschlecht } \\
\text { weiblich (\%) }\end{array}$ & 47,7 & 47,7 & $>0,999$ \\
\hline $\begin{array}{l}\text { Schulart } \\
\text { Gymnasium (\%) }\end{array}$ & 60,0 & 60,0 & $>0,999$ \\
\hline $\begin{array}{l}\text { Herkunft } \\
\text { In Deutschland geboren (\%) }\end{array}$ & 96,4 & 96,4 & $>0,999$ \\
\hline Subjektive Schulleistung & & & 0,798 \\
\hline Viel besser (\%) & 6,0 & 7,9 & \\
\hline Etwas besser (\%) & 34,1 & 32,4 & \\
\hline Etwa gleich (\%) & 48,9 & 45,3 & \\
\hline Etwas schlechter (\%) & 10,1 & 13,2 & \\
\hline Viel schlechter (\%) & 1,0 & 1,2 & \\
\hline $\begin{array}{l}\text { Subjektiver sozioökonomischer Sta- } \\
\text { tus (Skalenwert 1-10) }{ }^{\mathrm{a}}(\mathrm{M}, \mathrm{SD})\end{array}$ & $7,0( \pm 1,52)$ & $7,1( \pm 1,54)$ & 0,429 \\
\hline \multicolumn{4}{|c|}{ Persönlichkeitseigenschaften (Skalenwert 1-5) $(\mathrm{M}, \mathrm{SD})$} \\
\hline Offenheit & $3,5( \pm 1,01)$ & $3,5( \pm 0,95)$ & 0,207 \\
\hline Gewissenhaftigkeit & $3,2( \pm 0,94)$ & $3,3( \pm 0,90)$ & 0,792 \\
\hline Extraversion & $3,2( \pm 0,88)$ & $3,2( \pm 0,83)$ & 0,952 \\
\hline Verträglichkeit & $3,4( \pm 0,93)$ & $3,3( \pm 0,91)$ & 0,229 \\
\hline Neurotizismus & $3,0( \pm 0,94)$ & $2,9( \pm 0,91)$ & 0,548 \\
\hline $\begin{array}{l}\text { Sensation Seeking (Skalenwert 1-5) } \\
(M, S D)\end{array}$ & $2,0( \pm 1,09)$ & $1,9( \pm 1,07)$ & 0,689 \\
\hline
\end{tabular}

getrennt dargestellt für die Studienbedingungen, gibt - Tab. 1. Weder in der Ausprägung der Risikobereitschaft (Sensation seeking), in der subjektiven Schulleistung, im sozioökonomischen Status noch in der Persönlichkeitsstruktur konnten bedeutsame Unterschiede zwischen den Gruppen gefunden werden.

\section{Wissen und Einstellung}

Auf der Wissensebene zeigten sich systematische Unterschiede zwischen den Schülergruppen. Sie betrafen insbesondere die Wissensfragen zur Suchtentwicklung (IG: 58,9\%, KG 48,6\% korrekte Antworten, Pearson chi2(1) =3,92, $p=0,048)$ sowie Fragen zur Verbreitung des problematischen Medienkonsums im Jugendalter (IG: 35,0\%, KG: $13,4 \%$ korrekte Antworten, Pearson chi2 $(1)=28,44, \quad p<0,001)$. Die unterschiedlichen Ausprägungen der richtigen Antworten der Interventions- und Kontrollgruppe auf die Frage, welche ge- bedingung würde das nicht tun. In der Interventionsbedingung war es nur jeder Vierte $(23,7 \%)$, der trotz riskanten Konsums keine Hilfe in Anspruch nehmen würde.

\section{Mediennutzungsdauer: Spielen, Kommunikation, Unterhaltung sowie problematische Nutzung}

Die täglich durchschnittliche Computerspieldauer war bei der Interventionsgruppe signifikant niedriger im Vergleich zur Kontrollgruppe. Das traf sowohl für Schultage $(F(1,832)=6,45$, $p=0,011)$ als auch für schulfrei Tage $\mathrm{zu}$ $(\mathrm{F}(1,832)=4,06, p=0,044)$. Die durchschnittliche tägliche Kommunikation über soziale Medien war bei den Schüler*innen nach Workshop-Teilnahme mit 74 min rund 12 min kürzer als bei den Gleichaltrigen der Vergleichsgruppe, jedoch war der Unterschied nicht groß genug, um als signifikant detektiert werden zu können $(\mathrm{F}(1,832)=2,97$, $p=0,085)$. Am Wochenende oder an schulfreien Tagen hingegen chatteten die Workshop-Teilnehmer*innen signifikant kürzer (99 min) als die Schüler*innen der Kontrollgruppe (121 min, $\mathrm{F}(1,832)=4,08, p=0,044)$. An Schultagen verbrachte die Kontrollgruppe rund 19 min länger am Bildschirm mit Streaming $(\mathrm{F}(1,832)=5,83, p=0,016)$, an Wochenenden oder schulfreien Tagen fast eine halbe Stunde länger $(\mathrm{F}(1,832)=6,73$, $p=0,001)$. Die Nutzungszeiten veranschaulicht • Abb. 2.

Das Screening auf Grundlage der CIUS bezüglich einer exzessiv-dysfunktionalen Mediennutzung wies auf eine häufiger vorkommende problematische Nutzung in der Kontrollgruppe hin $(\mathrm{F}(1,832)=14,76, \quad p<0,001)$. Die Betrachtung der Verteilung der Skalenwerte zeigte, dass sich insbesondere mehr Schüler*innen mit niedrigen CIUS-Werten (Werte $<15$ ) in der Interventionsgruppe im Vergleich zur Kontrollgruppe fanden.

\section{Diskussion}

Die vorliegende kontrollierte Studie untersuchte die Auswirkungen einer schulbasierten Peer-to-peer-Präventionsmaßnahme zur Sensibilisierung für die Risi- 
Hier steht eine Anzeige.

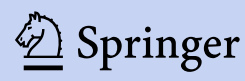




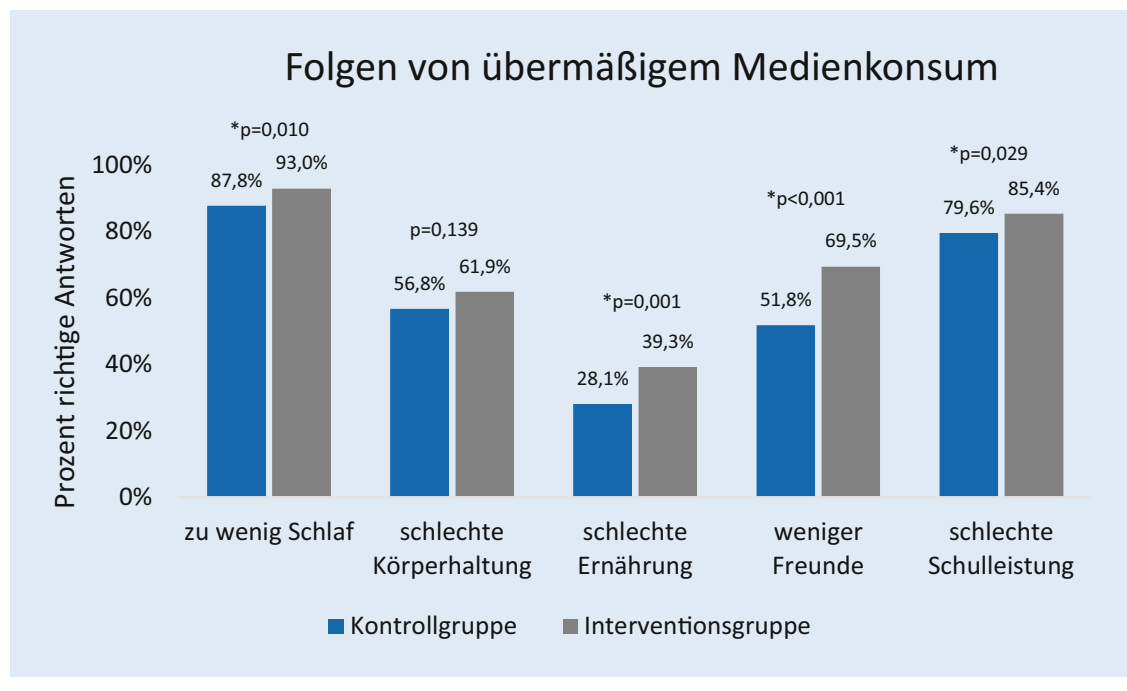

Abb. $1 \Delta$ Wissensitem: prozentualer Anteil der korrekten Antworten auf die Frage zu den gesundheitlichen Folgen von übermäßiger Mediennutzung, getrennt nach Gruppenzugehörigkeit. Statistisch signifikante Ergebnisse sind mit entsprechendem ${ }^{*} p$-Wert gekennzeichnet

ken der exzessiven Mediennutzung im Kindes- und Jugendalter an 834 Schüler*innen der Sekundarstufe I. Die Effektprüfung bezog sich auf das Wissen über exzessive Mediennutzung und ihre Folgen, die tägliche Computerspieldauer, Kommunikation über sozialen Medien und Streaming-Dauer sowie auf die problematische Mediennutzung.

Die Analysen zeigten eine Reihe von Interventionseffekten. Sie lieferten Hinweise darauf, dass sich ein Zuwachs an Wissen über Suchtentwicklung, zur Verbreitung der problematischen Mediennutzung im Jugendalter und zu sozialen und gesundheitlichen Risiken problematischer Nutzung einstellte. Interventionsschüler ${ }^{*}$ innen spielten weniger Computerspiele an Schultagen und an schulfreien Tagen, verbrachten weniger Zeit am Bildschirm mit Streaming-Angeboten und nutzten weniger soziale Medien an Wochenenden. In der Interventionsgruppe fanden sich zudem weniger Schüler*innen mit problematischer Mediennutzung. Positive Auswirkungen von schulbasierten Programmen über die Wissensebene hinaus wurden auch in den eingangs erwähnten Studien gefunden; die Befunde dieser Studie sind größtenteils vergleichbar $[2,19,20]$. Die Effekte auf der Verhaltensebene sind angesichts der kurzen Intervention, an der die Schüler*innen teilgenommen hatten, durchaus bemerkenswert.
Ein möglicher Erklärungsansatz basiert auf den theoretischen Annahmen zur Verhaltensänderung, wonach Menschen mit hoher Wahrscheinlichkeit eher ein erwünschtes Verhalten durchführen, wenn sie denken, dass dies ihrer Gesundheit nützt, wenn sie Druck durch das soziale Umfeld erfahren, sich entsprechend $\mathrm{zu}$ verhalten, und wenn sie die Entscheidungsmacht haben und sich in der Lage sehen, das Verhalten durchzuführen. Demnach könnte die Intervention deshalb Effekte erzielen, weil sie zur Selbstreflexion anregt, die Wahrnehmung der Gesundheitsrisiken übermäßiger Nutzung schärft und zu einem Wissenszuwachs über negative Folgen führt. Fundiertes Wissen kann zur Bildung von Überzeugungen führen, die Auswirkungen auf das eigene Verhalten haben, mit der Konsequenz, dieses ggf. korrigieren zu wollen. So kann ein verantwortungsvoller Umgang erlernt werden, der sich in geringeren Nutzungszeiten im Vergleich zu Kindern und Jugendlichen ohne ausgeprägtes Wissen widerspiegelt. Gleichwohl sich ein verantwortungsvoller Umgang nicht allein an der verbrachten Zeit messen lässt, sondern es ausschlaggebend ist, inwieweit es durch die Nutzungsweise zu Beeinträchtigungen kommt, hat die Mediennutzungsdauer die größte Vorhersagekraft für problematisches Verhalten und internetbezogene Störungen.
Kinder und Jugendliche, die seltener Zeit online verbringen, sind auch seltener von einer exzessiv-dysfunktionalen Nutzung betroffen. Die eingehende Prüfung von Interventionsmechanismen sollte $\mathrm{Ge}$ genstand zukünftiger Studien sein, um gezielt wirksame Programme für Kinder und Jugendliche aufzulegen.

Ein weiterer Erklärungsansatz für die Effektivität kann in der Projektstruktur liegen. Schwerpunktmäßig wurden die Kinder und Jugendlichen in den 2-mal 90-minütigen Workshops für die Gefahren der Mediennutzung sensibilisiert und auch das eigene Verhalten durch interaktive Übungen hinterfragt. Gleichwohl die Wirksamkeit von primär wissensbasierten Interventionen im Suchtbereich begrenzt zu sein scheint [13], wird bei der vorliegenden Intervention über das Individualverhalten hinaus das soziale Umfeld adressiert. Die Peers nahmen einen wichtigen sozialen Einfluss, indem sie die Inhalte der Intervention an jüngere Schüler*innen vermitteln und durch das Programm selbst zur Reflexion des eigenen Verhaltens angeregt wurden. Befunde einer Übersichtsarbeit zu Auswirkungen von Präventionsprogrammen zum Substanzkonsum beschreiben fähigkeitsfördernde Interventionen als vielversprechend, solche, die z. B. auf die Peer-Resistenz abzielen [17]. „Net-Piloten“ beinhaltet nicht nur die Wissensvermittlung, sondern bezieht auch mehrere Ebenen in der Schule ein, von der Unterbis zur Mittelstufe sowie mit dem Programm betraute Lehrkräfte. Die Peers stehen fortlaufend, mitunter über mehrere Schuljahre als Ansprechpartner*innen zur Verfügung und sind mit dem Thema vertraut. Die gesamte Klasse wird geschult, und somit werden soziale Normen im Setting Schule beeinflusst. Die soziale Beeinflussung wird durch den Befund zur Inanspruchnahme von Hilfe gestützt; Schüler*innen mit Interventionsteilnahme würden im Bedarfsfall eher Hilfe annehmen als Gleichaltrige ohne Teilnahme.

\section{Limitationen}

Im Gegensatz zu experimentellen Designs war die Zuordnung zur Studienbedingung in der vorliegenden Studie vor- 


\section{Dauer der Mediennutzung}

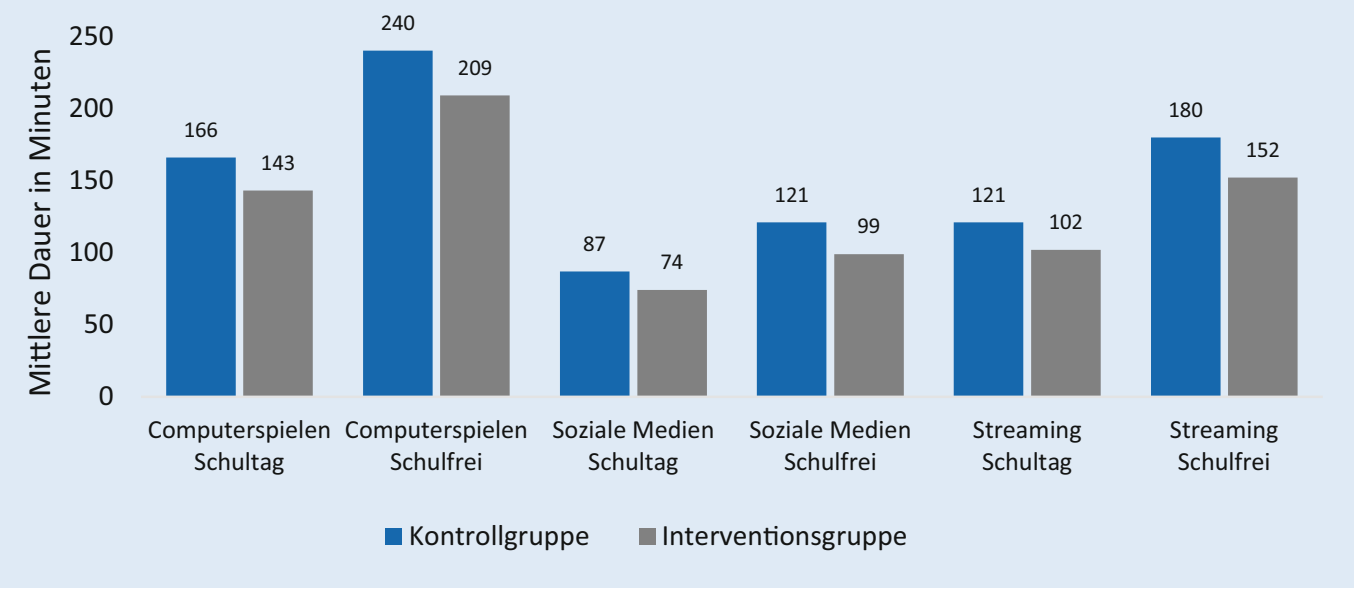

Abb. $2 \triangleleft$ Mittlere Dauer der Mediennutzung (Spiele, Kommunikation, Unterhaltung) an Schultagen und schulfreien Tagen in Minuten

gegeben; die Intervention fand bereits in der Vergangenheit statt. Um systematische Verzerrungen der Ergebnisse auch aufgrund fehlender Baseline-Werte zu reduzieren, wurde eine Substichprobe gezogen. Das Ziellag im Hervorbringen von „statistischen Zwillingen“, von dem einer mit und der andere ohne Interventionsteilnahme war. Die Zuordnungsprozedur wurde wiederholt durchgeführt und Ergebnisse auf Robustheit geprüft. Als Folge zeigte sich eine Übereinstimmung der Gruppen auch bezüglich stabiler Persönlichkeitseigenschaften. Die Strukturgleichheit ist relevant, stehen Persönlichkeitseigenschaften in direktem Zusammenhang mit dem Auftreten von internetbezogenen Störungen [5, 10]. Gleichwohl durch das Matching eine Strukturgleichheit geschaffen wurde, sind kausale Schlussfolgerungen jedoch nur äußerst eingeschränkt zu ziehen.

Trotz der Erfassung einer Reihe von Risikofaktoren ist eine Konfundierung des Zusammenhangs der Studienbedingung und des Mediennutzungsverhaltens durch eine oder mehrere nichterfasste Drittvariablen nicht auszuschließen. An dieser Stelle ist das soziale Umfeld zu nennen, Freunde außerhalb der Schule und/oder Eltern, die die Mediennutzung der Einzelnen bzw. des Einzelnen maßgeblich beeinflussen können. Auch wurden strukturelle Gegebenheiten wie die schulinternen Regeln, das "Schulethos“ zur Mediennutzung nicht als Störvariablen berücksichtigt.
Auch die Art der Datenerfassung stellt eine weitere Limitation der Studie dar, da keine objektive Messung herangezogen werden konnte. In Folge kann es, auch bedingt durch die Art der Erfassung der Mediennutzungsdauer, zu einer Fehleinschätzung beispielsweise in Form einer Überpathologisierung einer zum Lebensalltag junger Menschen gehörenden Tätigkeit kommen.

\section{Fazit für die Praxis}

- Das Angebot "Net-Piloten“ zur Sensibilisierung für die Risiken von exzessiver Mediennutzung (Spiele, Kommunikation, Unterhaltung etc.) wurde im Rahmen der Kampagne "Ins Netz gehen" (www.ins-netzgehen.de) der BZgA entwickelt und wird kontinuierlich weiterentwickelt.

- „Net-Piloten“ basiert auf dem Peerto-peer-Ansatz und involviert Kinder und Jugendliche im Alter von 12 bis 18 Jahren und adressiert neben der Individualebene organisationale Veränderungen in der Schule.

- Kinder und Jugendliche mit Teilnahme an dem Programm verfügten über ein höheres Wissen an negativen Konsequenzen exzessiver Nutzung im Vergleich zu denjenigen ohne Teilnahme.

- Kinder und Jugendliche mit Teilnahme an dem Programm zeigten geringere Nutzungszeiten digitaler
Anwendungen als die Vergleichsgruppe.

- Die Befunde stehen im Einklang mit früheren Studien zur Effektivität von schulbasierten Programmen zur Vermeidung von Internetabhängigkeit und Förderung der Medienkompetenz.

\section{Korrespondenzadresse}

\section{Dr. J. Hansen}

Institut für Therapie- und Gesundheitsforschung, IFT-Nord gemeinnützige $\mathrm{GmbH}$ Harmsstraße 2, 24114 Kiel, Deutschland hansen@ift-nord.de

Danksagung. Unser besonderer Dank gilt Andreas Pauly von update, Dr. Jana Janssen und Clemens Neumann für die Unterstützung bei der Studiendurchführung sowie allen Schulen für die Teilnahme.

Förderung. Die Studie wird von der Bundeszentrale für gesundheitliche Aufklärung im Auftrag des Bundesministeriums für Gesundheit gefördert.

\section{Einhaltung ethischer Richtlinien}

Interessenkonflikt. J. Hansen, R. Hanewinkel und M. Morgenstern geben an, dass kein Interessenkonflikt besteht. M. Goecke ist als Referatsleiterin für die Bundeszentrale für gesundheitliche Aufklärung tätig.

Für diesen Beitrag wurden von den Autoren keine Studien an Menschen oder Tieren durchgeführt. Für die aufgeführten Studien gelten die jeweils dort angegebenen ethischen Richtlinien.

Open Access. Dieser Artikel wird unter der Creative Commons Namensnennung 4.0 International Lizenz 
veröffentlicht, welche die Nutzung, Vervielfältigung Bearbeitung, Verbreitung und Wiedergabe in jeglichem Medium und Format erlaubt, sofern Sie den/die ursprünglichen Autor(en) und die Quelle ordnungsgemäß nennen, einen Link zur Creative Commons Lizenz beifügen und angeben, ob Änderungen vorgenommen wurden.

Die in diesem Artikel enthaltenen Bilder und sonstiges Drittmaterial unterliegen ebenfalls der genannten Creative Commons Lizenz, sofern sich aus der Abbildungslegende nichts anderes ergibt. Sofern das betreffende Material nicht unter der genannten Creative Commons Lizenz steht und die betreffende Handlung nicht nach gesetzlichen Vorschriften erlaubt ist, ist für die oben aufgeführten Weiterverwendungen des Materials die Einwilligung des jeweiligen Rechteinhabers einzuholen.

Weitere Details zur Lizenz entnehmen Sie bitte der Lizenzinformation auf http://creativecommons.org/ licenses/by/4.0/deed.de.

\section{Literatur}

1. Blackwell M, lacus $S$, King $G$ et al (2009) CEM: coarsened exact matching in stata. Stata J 9:524-546

2. Bonnaire C, Serehen Z, Phan O (2019) Effects of a prevention intervention concerning screens, and video games in middle-school students: Influences on beliefs and use. JBehav Addict 8:537-553

3. Dreier M, Wölfling K, Beutel ME (2014) Internetsucht bei Jugendlichen. Monatsschr Kinderheilkd 162:496-502

4. Feierabend S, Rathgeb T, Reutter T (2018) JIMStudie 2018 - Jugend, Information, Medien. Basisuntersuchung zum Medienumgang 12- bis 19Jähriger in Deutschland. Medienpädagogischer Forschungsverbund Südwest, Stuttgart

5. Geisel O, Lipinski A, Kaess M (2021) Non-substance addiction in childhood and adolescence-the Internet, computer games and social media. Dtsch Arztebl Int 118:14-22

6. Goodman E, Adler NE, Kawachi I et al (2001) Adolescents' perceptions of social status: development and evaluation of a new indicator. Pediatrics 108:E31

7. Grünbichler B, Pauly A, Niggestich A (2017) Net-Piloten Schulungsmanual. Peer-Projekt zur Prävention von Mediensucht. Bundeszentrale für gesundheitliche Aufklärung, Köln

8. Hansen J, Janssen J, Hanewinkel Ret al (2020) „NetPiloten " in der Praxis: Ergebnisse der Prozessevaluation eines schulbasierten Präventionsprogramms zur Vermeidung des exzessiven Computerspielund Internetgebrauchs im Jugendalter. Praev Gesundheitsf. https://doi.org/10.1007/s11553020-00808-7

9. Meerkerk GJ, Van Den Eijnden RJ, Vermulst AA et al (2009) The Compulsive Internet Use Scale (CIUS): some psychometric properties. Cyberpsychol Behav 12:1-6

10. Müller KW, Dreier M, Duven E et al (2017) Adding clinical validity to the statistical power of large-scale epidemiological surveys on Internet addiction in adolescence: a combined approach to investigate psychopathology and developmentspecific personality traits associated with Internet addiction. J Clin Psychiatry 78:e244-e251

11. Orth B, Merkel C (2020) Die Drogenaffinität Jugendlicher in der Bundesrepublik Deutschland 2019. Teilband Computerspiele und Internet.
In: BZgA-Forschungsbericht. Bundeszentrale für gesundheitliche Aufklärung, Köln

12. Paulus FW, Ohmann S, Von Gontard A et al (2018) Internet gaming disorder in children and adolescents: a systematic review. Dev Med Child Neurol 60:645-659

13. Poettgen S, Samkange-Zeeb F, Brand T et al (2015) Wie wirksam sind schulbasierte Interventionen zur Prävention und/oder Reduktion von Substanzkonsum bei Schülern/-innen in Grundund Sekundarschulen? Eine systematische Übersicht vorhandener Reviews. Gesundheitswesen 78:230-236

14. Rammstedt B, Kemper C, Klein MC et al (2013) Eine kurze Skala zur Messung der fünfDimensionen der Persönlichkeit: Big-Five-Inventory-10 (BFI-10) [A Short Scale for Assessing the Big Five Dimensions of Personality - 10 Item Big Five Inventory (BFI10). Methoden-Daten-Analysen 7:233-249

15. Rehbein F, Mößle T (2012) Risikofaktoren für Computerspielabhängigkeit: Wer ist gefährdet? Sucht 58:391-400

16. Stephenson MT, Hoyle RH, Palmgreen P et al (2003) Brief measures of sensation seeking for screening and large-scale surveys. Drug Alcohol Depend 72:279-286

17. Thomas R, Mclellan J, Perera R (2013) Schoolbased programs for preventing smoking. Cochrane Database Syst Rev. https://doi.org/10.1002/ 14651858.CD001293.pub3

18. Throuvala MA, Griffiths MD, Rennoldson $M$ et al (2019) School-based prevention for adolescent Internet addiction: prevention is the key. A systematic literature review. Curr Neuropharmacol 17:507-525

19. Walther B, Hanewinkel R, Morgenstern M (2014) Effects of a brief school-based media literacy intervention on digital media use in adolescents: cluster randomized controlled trial. Cyberpsychol Behav Soc Netw 17:616-623

20. Yang SY, Kim HS (2018) Effects of a prevention program for internet addiction among middle school students in South Korea. Public Health Nurs 35:246-255
MED UPDATE SEMINARE 2022

Pädiatrie Update 2022

15. Pädiatrie-Update-Seminar

06.-07. Mai 2022

Köln und Livestream

13.-14. Mai 2022

Berlin und Livestream

Pädiatrie Update 2023

16. Pädiatrie-Update-Seminar

21.-22. April 2023

Berlin und Livestream

28.-29. April 2023

Köln und Livestream

Wiss. Leitung:

Prof. Dr. Fred Zepp, Mainz

Prof. Dr. Stefan Wirth, Wuppertal

Prof. Dr. Reinhard Berner, Dresden

www.paediatrie-update.com

Auskunft für alle Update-Seminare: med update $\mathrm{GmbH}$ www.med-update.com

Tel.: 0611 - 736580

info@med-update.com 